\title{
A novel approach to assessing fetal tissue stiffness using virtual touch tissue quantification.
}

\author{
Xiao-Zhi Zheng ${ }^{1}$, Jing Wu ${ }^{1}$, Xu-Yan Tan ${ }^{2}$
}

${ }^{1}$ Department of Ultrasound, The First People's Hospital of Yancheng, Yancheng, ${ }^{2}$ Department of Ultrasound, Jiangsu Province Tumor Hospital, Nanjing, Jiangsu Province, P.R. China

\begin{abstract}
Aims: Virtual touch tissue quantification (VTTQ) has been widely used in adults, but its application in fetuses has not been reported. The purposes of this study were to describe the normal shear wave velocity (SWV) values of fetal brain, lung, and liver by VTTQ and to examine the clinical usefulness of this procedure in evaluation of gestational age-related SWV changes in the fetal brain, lung and liver. Material and methods: One hundred fetuses were enrolled in this study and were evaluated at 18 weeks (second trimester) and 35 weeks (third trimester) during pregnancy. The SWV was measured at the cerebral parenchyma, thalamus, cerebellum, choroid plexus, left lung, right lung, right and left lobe of the liver in each participant. Results: The SWV at the cerebral parenchyma were all significantly greater in the third trimesters than in the second trimesters $(3.29 \pm 1.05$ vs. $2.22 \pm 0.97 \mathrm{~m} / \mathrm{s}, \mathrm{p}<0.001)$, while the velocities at thalamus, cerebellum, choroid plexus, left lung, right lung, right lobe of the liver and left lobe of the liver did not differ between the second and the third trimesters $(\mathrm{p}>0.05)$. Moreover, the SWV at the cerebral parenchyma correlated significantly with gestational age $(r=0.47, p<0.001)$. Conclusions: VTTQ can provide numerical measurements of fetal brain, lung and liver stiffness and can effectively and objectively indicate gestational age-related changes in cerebral parenchyma stiffness by measuring SWV values.
\end{abstract}

Keywords: fetal tissues, stiffness, gestational age, shear wave velocity, virtual touch tissue quantification

\section{Introduction}

Ultrasound is currently the primary screening technique for imaging the fetus [1], playing an important role in the evaluation of the fetal organ development and detection of fetal organ abnormalities in utero. Virtual touch tissue quantification (VTTQ) is a promising new implementation of acoustic radiation force impulse ultrasound imaging, which provides numerical measurements [shear wave velocity (SWV) values] of tissue stiffness. Up to date, VTTQ has been widely used to evaluate the stiffness of superficial and deep tissues in adults, such as

Received 27.07.2015 Accepted 24.10.2015

Med Ultrason

2016, Vol. 18, No 1, 70-74

Corresponding author: Xu-Yan Tan,

Department of Ultrasound,

Jiangsu Province Tumor Hospital,

42 Baiziting Road, Nanjing 210009,

Jiangsu Province, P.R. China

Phone: $+86025-83283364$

Fax: $+86025-83283364$

E-mail: tanxuyanzxz@163.com the thyroid gland [2,3], breast [4,5], liver [6-9], kidneys $[6,10]$, prostate $[11,12]$, and other organs [6]. However, there are only two publications on the topic involving placental stiffness $[13,14]$ and the application of VTTQ in fetuses has not been reported. Fetal organs, especially the fetal brain, are rapidly developing and changing their appearances and structures week by week during pregnancy [15]. To our knowledge, the normal stiffness of fetal organs and their changes with age have remained unknown.

The purposes of this study were to describe the normal SWV values of fetal brain, lung, and liver by VTTQ and to examine the clinical usefulness of this procedure in the evaluation of gestational age-related SWV changes in the fetal brain, lung and liver.

\section{Material and methods}

\section{Study Population}

In this retrospective from 300 fetuses examined during pregnancy in our antenatal care center, 100 fetuses were randomly enrolled and were evaluated at 18 weeks (second trimester) and 35 weeks (third trimester). 
The inclusion criteria were the absence of any focal or diffuse diseases at any of the examined organs. Pregnant women with risk factors, such as diabetes mellitus, hypertension, congenital heart disease, cardiomyopathy, and endocrine diseases were excluded. The fetuses were evaluated by means of clinical and physical assessment, chromosome, ultrasonographic, and magnetic resonance imaging (MRI) examinations. The pregnant women were evaluated by means of clinical and physical assessment, laboratory data, electrocardiogram, ultrasonographic examinations, and MRI. The study was approved by the Human Research Ethics Committee of The Fourth Affiliated Hospital of Nantong University and signed informed consent was obtained from all the pregnant women.

\section{Equipment and examination}

A Siemens ACUSON S2000 US system (Siemens, Germany) equipped with convex probes (4C1) was used in this study. A mechanical index of 0.5 and tissue harmonic imaging of $4 \mathrm{MHz}$ were chosen. VTTQ was performed with the preliminary identification of a target region of interest (box with fixed dimension of $1 \times 0.5 \mathrm{~cm}$ ) on a conventional ultrasound image. Then, an acoustic push pulse was transmitted immediately on the right side of the region of interest where the SWV were calculated and expressed with a numerical value (meter/second, $\mathrm{m} / \mathrm{s}$ ) as a result of multiple measurements made for the same spatial location $[6,8]$.

For the brain study, two operators with 20 years experience in obstetrics and gynecology ultrasound performed three measurements at the cerebral parenchyma, thalamus, cerebellum and choroid plexus at the appropriate depth in the axial section (fig 1), respectively. The sampling frames were placed as much as possible perpendicular to the midline. For the lung and liver study, taking the stomach bubble and heart as a reference, respectively, the operators performed three measurements at the left lung and right lung, right lobe of the liver and left lobe of the liver (fig 1). The mean of three measurements of all organs was used for the statistical analysis.

\section{Reproducibility}

Intraobserver variability was assessed in 30 fetuses by repeating the measurements on two occasions ( 7 days

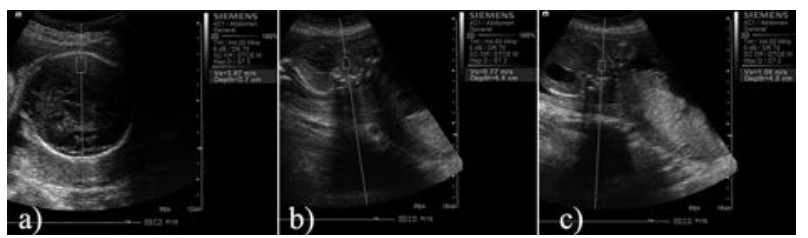

Fig 1. Shear wave velocity measurement at the cerebral parenchyma (a), right lung (b) and right lobe of the liver (c) with virtual touch tissue quantification. apart) under the same basal conditions (anterior dorsum position, $4 \mathrm{~cm}$ depth, $1 \times 0.5 \mathrm{~cm}$ box dimension). To test the interobserver variability, the measurements were successively performed on 30 fetuses by a second observer who was blind to the first observer's results. The reproducibility analysis was performed using intraclasss correlation coefficient and the mean relative difference with their $95 \%$ confidence intervals of the Bland-Altman plots.

\section{Statistical Analysis}

Data were expressed as the mean $\pm \mathrm{SD}$. The one-way analysis of variance (ANOVA) was employed to compare two measurements of SWV (once in each trimester) in the same fetus. A statistical comparison of differences between groups (different organs) was performed using an unpaired two tailed t test. The linear correlation analysis was used for determining the significance of correlations between SWV and gestational age. Agreements between intraobservers and interobservers in the measurement of SWV were assessed using intraclasss correlation coefficient and compared by Bland-Altman plots. Differences were considered significant at $p<0.05$. SPSS version 13.0 (SPSS, Chicago, IL, USA) was used for all statistical analysis.

\section{Results}

As shown in Table I, the SWV at the cerebral parenchyma in the second and third trimesters were all significantly greater than those at the thalamus $(p<0.05)$, and those at the cerebellum, choroid plexus, left lung, right lung, right and left lobe of the liver $(\mathrm{p}<0.01)$.

The SWV of the cerebral parenchyma were significantly greater in the third trimesters compared with the second trimesters $(p<0.01)$, while the velocities at the thalamus, cerebellum, choroid plexus, left lung, right lung, right lobe of the liver and left lobe of the liver did

Table I. Comparison of shear wave velocities $(\mathrm{m} / \mathrm{s})$ of different tissues between second trimesters and third trimesters

\begin{tabular}{lll}
\hline Organ & $\begin{array}{l}\text { Second } \\
\text { trimesters }\end{array}$ & $\begin{array}{l}\text { Third } \\
\text { trimesters }\end{array}$ \\
\hline Cerebral parenchyma & $2.22 \pm 0.97$ & $3.29 \pm 1.05^{*}$ \\
Thalamus & $1.57 \pm 0.91 \S$ & $1.76 \pm 0.87 \S$ \\
Cerebellum & $1.14 \pm 0.36^{* *}$ & $1.26 \pm 0.53^{* *}$ \\
Choroid plexus & $1.08 \pm 0.27^{* *}$ & $1.07 \pm 0.39^{* *}$ \\
Left lung & $0.94 \pm 0.28^{* *}$ & $1.05 \pm 0.49^{* *}$ \\
Right lung & $0.96 \pm 0.23^{* *}$ & $1.08 \pm 0.52^{* *}$ \\
Right lobe of the liver & $0.98 \pm 0.19^{* *}$ & $1.09 \pm 0.24^{* *}$ \\
Left lobe of the liver & $0.99 \pm 0.17^{* *}$ & $1.13 \pm 0.23^{* *}$ \\
\hline
\end{tabular}

$* \mathrm{P}<0.05$; ANOVA, compared to the values of second trimesters; $\S \mathrm{P}<0.05 ; * * \mathrm{P}<0.01$, unpaired $\mathrm{t}$ test, compared to the values of the cerebral parenchyma. 
Table II. Comparison of shear wave velocities $(\mathrm{m} / \mathrm{s})$ of different tissues between the fetuses' anterior dorsum and posterior dorsum position

\begin{tabular}{lll}
\hline Organ & $\begin{array}{l}\text { Anterior } \\
\text { dorsum }\end{array}$ & $\begin{array}{l}\text { Posterior } \\
\text { dorsum }\end{array}$ \\
\hline Cerebral parenchyma & $2.49 \pm 0.89$ & $2.51 \pm 0.97$ \\
Thalamus & $1.66 \pm 0.82$ & $1.69 \pm 0.92$ \\
Cerebellum & $1.22 \pm 0.39$ & $1.23 \pm 0.43$ \\
Choroid plexus & $1.08 \pm 0.28$ & $1.05 \pm 0.37$ \\
Left lung & $0.96 \pm 0.38$ & $1.01 \pm 0.47$ \\
Right lung & $1.02 \pm 0.38$ & $1.03 \pm 0.44$ \\
Right lobe of the liver & $1.07 \pm 0.23$ & $1.06 \pm 0.26$ \\
Left lobe of the liver & $1.09 \pm 0.19$ & $1.11 \pm 0.22$ \\
\hline
\end{tabular}

not differ between the second and the third trimesters $(\mathrm{p}>0.05)$.

As shown in table II, there was no difference in the measurements of SWV from anterior or posterior dorsum position of the fetus.

The Pearson correlation analysis showed that the SWV at the cerebral parenchyma correlated significantly with gestational age $(r=0.47, \mathrm{p}<0.001)$. The linear regression equation was $\mathrm{y}=0.01 \mathrm{x}+0.96\left(\mathrm{R}^{2}=0.22, \mathrm{p}<0.001\right)$, where $\mathrm{x}$ and $\mathrm{y}$ represent gestational age (days) and the SWV, respectively (fig 2). However, there was no significant correlation between the SWV and gestational age at the thalamus, cerebellum, choroid plexus, left lung, right lung, right lobe of the liver and left lobe of the liver $(\mathrm{r}=0.09, \mathrm{r}=0.06, \mathrm{r}=0.01, \mathrm{r}=0.015, \mathrm{r}=0.06, \mathrm{r}=0.17$ and $\mathrm{r}=0.2$, respectively, $\mathrm{p}>0.05$ )

As shown in figure 3, inter and intra observer agreement in the measurement of SWV was fairly good: the intraclass correlation coefficient was $0.92(0.84-0.96)$, 0.94 (0.86-0.99) for inter and intra observer, respectively.

\section{Discussions}

The results presented here indicate that VTTQ can provide numerical measurements of the fetal brain, lung and liver stiffness and can effectively and sensitively indicate the stiffness changes at cerebral parenchyma, which provide a new approach to assessing fetal cerebral parenchyma development.

The change in the stiffness of fetal organs is a useful sign for detecting their abnormalities. However, the relationship between fetal organs' stiffness and gestational age has seldom been described in the literature, perhaps because of a lack of effective and objective evaluation strategies. Acoustic radiation force impulse imaging is a new ultrasound imaging modality for evaluating the tissue stiffness, which make objective analysis of tissue stiffness possible. This imaging technique can produce


Fig 2. Correlation between shear wave velocity and gestational age at the cerebral parenchyma.
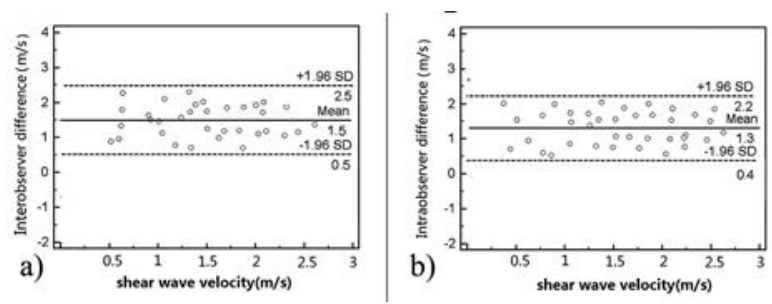

Fig 3. Bland-Altman graphs displaying differences against average values of shear wave velocity between inter/intra observer 1 and inter/intra observer 2 . The thick line represents mean difference and the dashed lines represent the limits of agreement; a) interobserver difference; b) intraobserver difference.

localized displacements in a "pushed" region of interest $[6,16,17]$, by short-duration acoustic radiation forces, where shear waves are generated without the need for external compression [18]. The SWV can be easily measured by VTTQ without special preparations. In our study, the SWV could be reproducibly quantified at all regions of interest in all participants. The intraobserver and interobserver variability of the SWV measurements were all within an acceptable scope (intraclass correlation coefficient $>0.9$ )

Fetal organs develop rapidly during pregnancy. All the fetal organs have been basically formed in the first trimester. However, unlike other organs, the central nervous system continues to develop during the second and third trimesters, although it has developed remarkably in the first trimester. Because it is quite difficult to observe detailed structures of fetal organs and their function changes in the first trimester, we chose the fetuses in the second and third trimesters as our study objects.

Our study showed that the stiffness of the cerebral parenchyma was significantly greater in the third trimesters compared with the second trimesters, and these values correlated significantly with gestational age. This finding is related to a remarkable development of the cerebral parenchyma in the third trimesters [15,19-21]. However, the stiffness of thalamus, cerebellum, choroid plexus, left lung, right lung, right lobe of the liver, and left lobe of the liver did not differ between the second and the third 
trimesters and there is no relationship between the stiffness and gestational age for these organs. We have not yet found reasons to explain this phenomenon. Maybe, VTTQ is not sensitive enough to detect their stiffness differences between the second trimester and the third trimester.

Our results manifested that the fetuses have different organ SWV values rather than a uniform value, and the SWV values at the cerebral parenchyma increase with gestational age. These findings are consistent with fetal organ development law. This reminds us that gestational age should be taken into consideration when using SWV as an indicator of a differential diagnosis of cerebral parenchyma.

Although VTTQ can potentially be an important screening tool for fetal development, several limitations should be mentioned. First, it is not sensitive enough because shear wave propagation is extremely short. Secondly, the detection depth (maximum, $5.5 \mathrm{~cm}$ ) of VTTQ is limited, the box dimension of the region of interest is fixed, and it is very sensitive to movement artifacts. These limitations may become obstacles to the extensive application of this new technology [6,22]. Thirdly, the fetuses' population examined was limited. New data need to be collected in subsequent studies. In addition, whether VTTQ has effects on fetal development requires further research.

\section{Conclusion}

In this study, we evaluated the usefulness of VTTQ for the assessment of fetal brain, lung and liver stiffness. Our results showed that fetal brain, lung and liver have different stiffness, and the SWV at the cerebral parenchyma correlated significantly with gestational age. VTTQ can effectively and objectively indicate gestational agerelated changes in cerebral parenchyma stiffness which provides a new approach to assessing fetal cerebral parenchyma development. Although VTTQ is feasible in pregnancy, further studies are required.

\section{Acknowledgements}

The authors gratefully acknowledge the technical assistance and helpful discussions with Wang JW, Huang XQ, Fan GX at the Department of Ultrasound in the First People's Hospital of Yancheng, Jiangsu Province, P.R.China.

\section{Conflict of interest: none}

\section{References}

1. Girard N, Chaumoitre K, Chapon F, Pineau S, Barberet $\mathrm{M}$, Brunel H. Fetal magnetic resonance imaging of ac- quired and developmental brain anomalies. Semin Perinatol 2009; 33: 234-250.

2. Hou XJ, Sun AX, Zhou XL, et al. The application of virtual touch tissue quantification (VTQ) in diagnosis of thyroid lesions: A preliminary study. Eur J Radiol 2013; 82: 797-801.

3. Zhang YF, Xu HX, He Y, et al. Virtual touch tissue quantification of acoustic radiation force impulse: a new ultrasound elastic imaging in the diagnosis of thyroid nodules. PLoS One 2012; 7: e49094.

4. Jin ZQ, Li XR, Zhou HL, et al. Acoustic radiation force impulse elastography of breast imaging reporting and data system category 4 breastlesions. Clin Breast Cancer 2012; 12: 420-427.

5. Bai M, Du L, Gu J, Li F, Jia X. Virtual touch tissue quantification using acoustic radiation force impulse technology: initial clinical experience with solid breast masses. J Ultrasound Med 2012; 31: 289-294.

6. Gallotti A, D'Onofrio M, Pozzi Mucelli R. Acoustic Radiation Force Impulse (ARFI) technique in ultrasound with Virtual Touch tissue quantification of the upper abdomen. Radiol Med 2010; 115: 889-897.

7. Zhang P, Zhou P, Tian SM, Qian Y, Deng J, Zhang L. Application of acoustic radiation force impulse imaging for the evaluation of focal liver lesion elasticity. Hepatobiliary Pancreat Dis Int 2013; 12: 165-170.

8. Gallotti A, D'Onofrio M, Romanini L, Cantisani V, Pozzi Mucelli R. Acoustic Radiation Force Impulse (ARFI) ultrasound imaging of solid focal liver lesions. Eur J Radiol 2012; 81: 451-455.

9. Monti L, Manco M, Lo Zupone C, et al. Acoustic radiation force impulse (ARFI) imaging with Virtual Touch Tissue Quantification in liver disease associated with cystic fibrosis in children. Radiol Med 2012; 117: 1408-1418.

10. Stock KF, Klein BS, Vo Cong MT, et al. ARFI-based tissue elasticity quantification in comparison to histology for the diagnosis of renal transplant fibrosis. Clin Hemorheol Microcirc 2010; 46: 139-148.

11. Zheng XZ, Ji P, Mao HW, et al. A novel approach to assessing changes in prostate stiffness with age using virtual touch tissue quantification. J Ultrasound Med 2011; 30: 387-390.

12. Zheng X, Ji P, Mao H, Hu J. A comparison of virtual touch tissue quantification and digital rectal examination for discrimination between prostate cancer and benign prostatic hyperplasia. Radiol Oncol 2012; 46: 69-74.

13. Kılıç F, Kayadibi Y, Yüksel MA, et al. Shear wave elastography of placenta: in vivo quantitation of placental elasticity in preeclampsia. Diagn Interv Radiol 2015; 21: 202-207.

14. Quibel T, Deloison B, Chammings F, et al. Placental elastography in a murine intrauterine growth restriction model. Prenat Diagn 2015; 35: 1106-1111.

15. Pooh RK. Normal anatomy by three-dimensional ultrasound in the second and third trimesters. Semin Fetal Neonatal Med 2012; 17: 269-277.

16. Nightingale K, Soo MS, Nightingale R, Trahey G. Acoustic radiation force impulse imaging: in vivo demonstration of clinical feasibility. Ultrasound Med Biol 2002; 28: 227-235. 
17. D’Onofrio M, Gallotti A, Martone E, Pozzi Mucelli R. Solid appearance of pancreatic serous cystadenoma diagnosed as cystic at ultrasound acoustic radiation force impulse imaging. JOP 2009; 10: 543-546.

18. Nightingale K, Bentley R, Trahey G. Observations of tissue response to acoustic radiation force: opportunities for imaging. Ultrason Imaging 2002; 24: 129-138.

19. FuYC, Zhang CB, Mao CW, et al. Relationship between gestational age and apparent diffusion coefficient values in different regions of fetal brain from middle to late trimester. Zhonghua yi xue za zhi 2013; 93: 2463-2466.
20. Yang L, Chen L, Qiu X, et al. Fetal cerebral lobes development between 20 and 28 weeks gestational age: a postmortem MR study. Int J Dev Neurosci 2014; 32: 23-27.

21. Imanishi M, Tanikake T, Kyoi K, Utsumi S. Neuroradiological study of human brain in the fetal period. Neurol Res 1988; 10: 40-48.

22. Yamanaka N, Kaminuma C, Taketomi-Takahashi A, Tsushima Y. Reliable measurement by virtual touch tissue quantification with acoustic radiation force impulse imaging: phantom study. J Ultrasound Med 2012; 31: 1239-1244. 